\title{
CONFISERIES ET DIPLOMATIE DANS LES COLONIES DE LA RÉPUBLIQUE DE GÊNES SUR LA MER NOIRE À LA FIN DU MOYEN ÂGE*
}

\author{
Rafał Hryszko \\ Uniwersytet Jagielloński w Krakowie
}

\begin{abstract}
CONFECTIONERY AND DIPLOMACY IN THE COLONIES OF THE REPUBLIC OF GENOA BY THE BLACK SEA TOWARDS THE END OF THE MIDDLE AGES
\end{abstract}

The article consists of two basic parts. The first part presents a characteristics of the diplomatic activities of Caffa, the main colony of the Republic of Genoa on the southern coast of the Crimean Peninsula in the 1380s. Of special significance in that domain were contacts with the Tatar emirs of Crimea who represented the Khan of the White Horde - Tokhtamysh. Those contacts led to a series of treaties (in 1381, 1382 and 1387), which resulted in the Genoese strengthening their position on the southern coast of Crimea. Moreover, in place of existing trading factories an overseas territory was established in southern Crimea, which belonged directly to the Republic of Genoa. In the second part of the article the author presents the role and significance of confectionery in the above mentioned diplomatic activities, on the basis of an analysis of the contents of the three oldest fiscal books of the Caffa massaria (tax office) (ASG, MC, room 34, 590/1225, 1226 and 1226-1) from the years: 1374-1375, 1381-1382 and 1386-1387. Confectionery appeared during feasts that rulers of Caffa held for foreign envoys. Sweets were often served with wine during snacks known as collationes. Finally the author proposes a hypothesis that such a form of refreshments in the world of Genoese diplomacy by the Black Sea was practised since at least 1375 , though under a different name.

Key words: the Republic of Genoa, Caffa, diplomacy, confectionery, $14^{\text {th }}$ century.

Słowa kluczowe: Republika Genui, Caffa, dyplomacja, słodycze, XIV wiek.

Mots-clés: République de Gênes, Caffa, diplomatie, confiseries, XIVe siècle.

* La présentation du contenu de cet article a eu lieu lors du Colloque International: Table et diplomatie à l'échelle du monde, Paris, le 2-5 novembre de 2016 organisé par l'Institut Européen d'Histoire et des Cultures de l'Alimentation.

Adresse de correspondance: rafal.hryszko@uj.edu.pl 
Ala fin du XIII ${ }^{\mathrm{e}}$ s., le littoral de la Mer Noire fut couvert par un réseau de comptoirs de la République de Gênes. Les plus importants furent implantés à Caffa (Théodosie ou Féodosie) - en Crimée, à Tana (à l'embouchure du Don), à Kilia et Licostomo (dans le delta du Danube), à Bielogorod (Moncastro, sur le Dniestr), à Trébizonde sur les côtes septentrionales de l'Asie Mineure et à Constantinople (Pera-Galata) ${ }^{1}$. Au début de leur existence, ils servirent d'intermédiaire dans l'importation en Europe de marchandises venues du Levant (épices, soie, coton et autres) ${ }^{2}$.

$\mathrm{Au}$ milieu du XIV ${ }^{\mathrm{e}}$ s., le commerce de Gênes fut touché de plein fouet par la crise économique causée par l'affaiblissement des relations avec l'Extrême-Orient. Dans cette situation, les Génois furent amenés non seulement à chercher de nouvelles sources d'échanges commerciaux mais, avant tout, à sauvegarder leur position politique dans la région de la Mer Noire ${ }^{3}$.

A la suite d'un jeu diplomatique complexe et de différentes actions politiques, les commerçants de Gênes réussirent à reprendre le contrôle des côtes sud de la Crimée avec Cembalo (vers 1344), Soldaïa (en 1365) et le territoire dénommé Gothie ${ }^{4}$.

Dans les années 60 et 70 du XIV ${ }^{\mathrm{e}}$ s., l'émir tartare Mamaï tenta en vain de reconquérir ces territoires. Ces plans furent anéantis par les défaites qui lui avaient

1 M. Małowist, Kaffa - kolonia genueńska na Krymie i problem wschodni w latach 1453-1475, Warszawa 1947; S.P. Karpov, Ital'janskie morskie respubliki i Juznoe Pricernomor'e v XIII-XV vv.: problemy torgovli, Moskva 1990; M. Balard, La Romanie génoise (XII ${ }^{e}$-début du XVe siècle), RomaGenova 1978; G. Jehel, Les Génois én Mediterranee occidentale (fin XI ${ }^{e}$-début XIVtesiècle), Paris 1993; R.S. Lopez, Storia delle colonie genovesi nel Mediterraneo, Genova 1996; D. Quirini-Popławska, Włoski handel czarnomorskimi niewolnikami w późnym średniowieczu, Kraków 2002; R. Hryszko, Z Genui nad Morze Czarne. Z kart genueńskiej obecności gospodarczej na pótnocno-zachodnich wybrzeżach Morza Czarnego u schytku średniowiecza, Kraków 2004; A. Ste1lo, Grenzerfahrung. Interaktion und Kooperation im spätmittelalterlichen Schwarzmeerraum, Karlsruhe 2012, http://ubt.opus.hbz-nrw. de/volltexte/2012/753 [accès: 8.09.2017]; E. Khvalkov, The Colonies of Genoa in the Black Sea Region: Evolution and Transformation, New York-London 2017.

2 F. Balducci Pegolotti, La pratica della mercatura, ed. A. Evans, Cambridge, Mass. 1936, p. 21-23; W. Heyd, L'histoire du commerce du Levant au Moyen Age, vol. II, Paris 1936, p. 176-177; R.H. Bautier, Les relations économiques des Occidentaux avec les pays d'Orient au Moyen Age. Points de vue et documents [dans:] Sociétés et compagnies de commerce en Orient et dans l'Océan Indien, Actes du $8^{e}$ Colloque international d'Histoire maritime, Beyrouth 1966, Paris 1970, p. 315-316; R.S. Lopez, L'extrême frontière du commerce medieval, „Le Moyen Âge” vol. jubilaire: 1963, p. 479-480; idem, In quibuscumque mondi partibus [dans:] Miscellanea di storia italiana e mediterranea per Nino Lamboglia, Genova 1978, p. 345-354; M. Balard, Precursori di Cristoforo Colombo: i Genovesi in Estremo Oriente nel XIV secolo [dans:] Atti del Convegno Internazionale di Studi Colombiani (Genova 13-14 ottobre 1973), Genova 1974, p. 149-161; idem, La Romanie..., s. 136, 202-204, 684-686; idem, Gênes et la mer Noire (XIII ${ }^{e}-X V^{e}$ siècle), „Revue Historique” 1983, 270, p. 31-40; E. A shtor, Il volume del commercio levantino di Genova nel secondo Trecento [dans:] Civico Istituto Colombiano, Studi e Testi, p. 2, Serie storica a cura di G. Pistarino, Saggi e documenti, vol. I, Genova 1978, p. 391-432; S.P. Karpov, op. cit., p. 112-113.

3 B.Z. Kedar, Mercanti in crisi: Venezia e Genova, Roma 1981.

4 N. Iorga, Notes et extraits pour servir à l'histoire des croisades au XVé siècle, ,Revue de l'Orient latin", t. IV, Paris 1896, p. 32; B. Grekow, A. Jakubowski, Ztota Orda i jej upadek, Warszawa 1953, p. 223; M. Balard, La Romanie..., p. 457; V. Ciocîltan, Mongoli şi Marea Neagră în secolele XIIIXIV. Contribuţia Cinghizhanizilor la transformarea bazinului pontic în placă turnantă a comerţului euro-asiatic, Bucureşti 1998, p. 206. 
été infligées par Dimitri Donskoï, grand prince de Moscou et par Tokhtamych, khan de la Horde Blanche.

En 1380, les Génois se virent contraints à composer avec le victorieux Tokhtamych. C'était aussi une chance pour garder durablement les territoires conquis sur les côtes sud de la Crimée 5 .

Quelles actions entreprit la diplomatie de Caffa dans le nouvel environnement tel que celui constitué dans les années $80 \mathrm{du} \mathrm{XIV}^{\mathrm{e}} \mathrm{s}$. à l'égard des gouverneurs tartares de la Crimée, représentant le victorieux Tokhtamych? Quelles méthodes mirent-ils en oeuvre? Quel rôle y jouèrent les confiseries? - ce sont les questions qui nous guideront dans nos considérations.

Dans les recherches de la réponse aux questions ainsi formulées, il s'est avéré indispensable de puiser aux archives et, notamment, aux registres de la Massaria de Caffa des années 1374-1375, 1381-1382 et 1386-13876.

Je me suis basé sur des matériaux de comparaison soit sur quatre registres de la Trésorerie de Péra de la charnière des XIV et $\mathrm{XV}^{\mathrm{e}}$ siècles ${ }^{7}$ et huit registres successifs de la Trésorerie - de la Massaria de Caffa des années 1410 à 1446 dont des extraits ont été aimablement mis à ma disposition par dr Annika Stello de Badischen Landesbibliothek de Karlsruhe (ce dont je tiens ici à la remercier).

Quant à la première question posée, il y a lieu de constater que les défaites essuyées par Mamaï ont facilité la tâche de la diplomatie de Gênes et l'établissement des relations avec Tokhtamych. Ainsi, Cerchez, représentant du khan de la Horde Blanche qui était venu en Crimée, a non seulement opéré un changement de la politique, généralement défavorable aux Génois, menée jusqu'à cette époque par les gouverneurs tartares de la Crimée 9 . En novembre 1380 (27.XI), il a aussi conclu un traité bilatéral dans lequel les Tartares ont reconnu la suprématie de la République de Gênes sur la côte sud de la Crimée, de Cembalo à Soldaï ${ }^{10}$. Les parties se garantissaient mutuellement la liberté du commerce et la sécurité. Chaque mois, un fonction-

5 A.N. Nasov, Mongoly i Rus, Moskva-Leningrad 1940, p. 126; B. Grekov, A. Jakubowski, op. cit., p. 233-237; B. Spuler, Die Goldene Horde. Die Mongolien in Russland (1223-1502), Wiesbaden 1965, p. 126; V. Ciocîltan, Restauraţia Hoardei de Aur şi tratatele tătaro-genovese din anii 1380-1387, „Revistă Istorică” 1990, 1, n. 6, p. 576; idem, Mongoli..., p. 207-208.

6 Archivio di Stato di Genova (plus loin: ASG), Banco di San Giorgio, Massaria Caffae (plus loin: MC), sala 34, 590/1225, 1226, 1226-1. A voir aussi: A.L. Ponomarev, Putevoditel' po rukopisi massarii Kaffy $1374 \mathrm{~g}$. Liber massariae Caffae tempore regiminis egregii viri domini Iuliani de Castro consulis Caffae MCCCLXXIV nunc indicatus et a pluribus mendis purgatus [dans:] Prichernomorie $v$ srednie veka, vypusk 6, Moskva 2005, p. 41-138.

ASG, Banco di San Giorgio, Massaria Peire (plus loin: MP), sala 34, 590/1303-1305. Les fragments des livres de comptes de Péra, Caffa et Famaguste ont été publié par N. Iorga, op. cit., p. 25-118.

8 ASG, MC, sala 34, 590/1227-1235, 1264.

9 R. Grousset, L'Empire des steppes, Paris 1939, p. 485; V. Ciocîltan, Mongoli..., p. 210, 212; idem, Reichspolitik und Handel: Die tatarisch-genuesischen Verträge von 1380-1387, „Il Mar Nero. Annuario di archeologia e storia" 1994, 1, p. 262-264.

10 V. Ciocîltan, Mongoli..., p. 212; A.A. Vasiliev, The Goths in the Crimea, Cambridge 1936, p. 180-181; I.S. Pioro, Krymskaja Gotija: Očerki etničeskoj istorii naselenija Kryma v pozdnerimskij period i rannee srednevekovie, Kiev 1990, p. 86-87. 
naire tartare devait venir à Caffa pour prélever le droit de douane au profit du khan à concurrence de 3 à $5 \%$ de la valeur des marchandises ${ }^{11}$.

Quelques mois après la signature du premier traité, en février 1381 (24.II), fut signé un nouvel accord au contenu similaire. Celui-ci ne faisait que refléter les changements qui étaient intervenus dans la partie tartare de la Crimée. Cerchez fut remplacé dans ses fonctions de gouverneur de la Crimée par Ilias (Elias), fils de Koutloug Beg, dignitaire tartare influent ${ }^{12}$. Il découle des inscriptions dans les registres de la Trésorerie de 1381 qu'en août et en novembre de cette année, il fut solennellement accueilli à $\mathrm{Caffa}^{13}$.

Au début de l'année 1382, Koutloug Beg en personne vint en Crimée ${ }^{14}$. Comme le suppose Virgil Ciocîltan, en mars de cette même année fut conclu un troisième accord réglant les relations entre les Génois et les Tartares. Hélas, les stipulations de celui-ci ne furent pas sauvegardées et ne sont pas connues de nos jours ${ }^{15}$.

En même temps, les Génois maintenaient de bonnes relations avec Tokhtamych. En 1382, à deux reprises (en février et en novembre), une députation fut envoyée à sa cour avec des cadeaux somptueux.

Comme l'observe, à juste titre, Annika Stello, c'étaient les autorités de Caffa génoise qui initièrent les mesures susmentionnées. C'étaient elles qui tenaient à maintenir les meilleures relations possibles avec les Tartares. En effet, en dépendait l'existence des colonies les plus importantes que Gênes possédait dans la région de la Mer Noire c'est-à-dire en Crimée.

Tout porte à croire que, pendant trois années consécutives, les deux parties maintiennent le status quo. Cependant, en 1385, les habitants tartares de la Gothie s'insurgent contre l'administration génoise et, une année plus tard, cette rébellion se transforme en une guerre entre les Génois et les Tartares ${ }^{16}$. Après les succès ini-

11 Le texte du traité de 27 novembre de 1380 a été publié par S. de Sacy, Pièces diplomatiques tirées des Archives de la Républiques du Gênes [dans:] Notices et extraits des manuscrits de la Bibliothèque du Roi, vol. XI, Paris 1827, p. 51-58; C. Desimoni, Trattato dei Genovesi col chan dei Tartari nel 1380-1381, scritto in lingua volgare, „Archivio Storico Italiano” 1887, 20, p. 161-165; N. Iorga, op. cit., p. 12, 17; B. Spuler, op. cit., p. 114-115, 120-121; E. Bas so, Il „,bellum de Sorcati” ed i trattati del 1380-87 tra Genova e l'Orda d'Oro, „Studi Genuensi”, n. s., 1990, 8, p. 12-13; V. Ciocîltan, Mongoli..., p. 209-210, 214; idem, Restauraţia ..., p. 579-580.

12 W. Heyd, op. cit., t. II, s. 205; V. Ciocîltan, Mongoli..., s. 213.

13 ASG, MC, sala 34, 590/1226, fol. 63v (3.VIII.1381), 159v (27.XI.1381), 294r (5.XI.1381).

14 ASG, MC, sala 34, 590/1226, fol. 65v (20.I.1382); N. Iorga, op. cit., p. 15-16; E. Basso, Il „,bellum de Sorcati”..., p. 13-14; V. Ciocîltan, Mongoli..., p. 214-215, 218; idem, Restauraţia..., p. 584-585; idem, Reichspolitik..., p. 265-268.

15 V. Ciocîltan, Reichspolitik..., p. 270-276.

16 N. Iorga, op. cit., p. 16-17; G. Pistarino, I Gin dell'Oltremare [dans:] Civico Istituto Colombiano, Studi e Testi, Serie storica a cura di G. Pistarino, Genova 1988, p. 214; M. Balard, La Romanie..., p. 157-159, 161; idem, Les Génois et les régions bulgares au XIVE siècle, „Byzantino-bulgarica” 1981, 7, p. 93-94; Ş. Papacostea, „Quod non iretur ad Tanam”. Un aspect fondamental de la politique génoise dans la mer Noire au XIV siècle, „Revue des Études Sud-Est Européenes” 1979, 17, n. 2, p. 39; E. Basso, Il „, bellum de Sorcati”..., p. 12-13; V. Ciocîltan, Mongoli..., p. 220-221; O.S. Mavrina, Nekotoryye aspekty genuezsko-tatarskikh otnosheniya v XIV veke, „Skhodoznavstvo” 2005, 29-30, p. 89-99; A.L. Ponomarev, «Solkhatskaya voyna» i «imperator» Bek Bulat [dans:] Materialy vtoroi 
tiaux des Génois, les Tartares remportent la victoire qui pousse les autorités de Caffa à chercher des voies pacifiques pour régler le conflit ${ }^{17}$.

De juillet 1386 à septembre 1387, plusieurs députations génoises sont envoyées à Solkhat. Les négociations (soutenues par des cadeaux généreux) permettent de mettre fin au conflit ${ }^{18}$.

En août 1387 (12.VIII), un nouveau traité intervient entre les Génois et les Tartares. A l'instar des documents précédents, y sont confirmés les accords antérieurs; ceux-ci s'accompagnent d'une déclaration de volonté de poursuivre les bonnes relations entre les parties ${ }^{19}$.

Ainsi, à la suite des traités successifs, les Génois renforcent définitivement leur position sur le littoral sud de la Crimée. De plus, les comptoirs isolés dans le sud de la Crimée sont remplacés par un territoire d'outre-mer appartenant directement à la République de Gênes.

\section{$* * *$}

Les succès dont ont été couronnées les actions entreprises par les autorités de Caffa, n'auraient pas été possibles si les Génois n'avaient pas eu recours aux méthodes diplomatiques pertinentes. En premier lieu, c'étaient des représentants dépêchés à plusieurs reprises à Solkhat - siège des gouverneurs successifs du khan Tokhtamych et au khan lui-même. En second lieu, un rôle majeur incomba aux différents types de réceptions organisées à Caffa pour honorer les gouverneurs de Solkhat ou les députés tartares. Les nombreux cadeaux somptueux que les Génois transmettaient aux Tartares n'y étaient non plus pour rien. Et, dans ce contexte, quelle place incombait-elle aux confiseries?

Dans les documents analysés de la Massaria de Caffa du XIV s., les confiseries apparaissent déjà plus tôt que pendant la période concernées par nos études, c'està-dire au milieu des années $70 \mathrm{du} X \mathrm{XIV} \mathrm{e}^{\mathrm{s}} \mathrm{s}$. Bien que la première mention concernant la consommation de confiseries est datée du novembre $1374^{20}$, l'on parle de la présence de confiseries dans le domaine diplomatique seulement à partir de l'année suivante. Dans les documents de la Trésorerie de Caffa il est noté qu'en avril 1375, au

Mezhdunarodnoy nauchnoy konferentsii «Politicheskaya i sotsial'no-ekonomicheskaya istoriya Zolotoy Ordy", posvyashchennoy pamiati M.A. Usmanova, Kazan', 29-30 marta 2011 g., Kazan' 2011, p. 18-21; Ya.V. Pilipchuk, Voyny v zolotoordynskom Krymu: realii i vymysel (40-ye gg. XIV v.-10-ye gg. XV v.), „Parabellum novum” 2017, nr 7(40), p. 55-69.

17 M. Balard, La Romanie..., s. 157-159; idem, Les Génois et les régions bulgares..., s. 93-94; E. Basso, Il ,,bellum de Sorcati”..., s. 12-13; idem, „De rebus castri Illicis et alia”. Genova, la Moldavia e la Valacchia fra cooperazione e contrasto nel secondo Quattrocento [dans:] Italia e Romania. Due popoli e due storie a confronto (secc. XIV-XVIII), a cura di S. Graciotti, Firenze 1997, s. 85; V. Ciocîltan, Mongoli..., s. 220-221.

18 E. Basso, Il ,, bellum de Sorcati”..., p. 15; M. Balard, La Romanie..., p. 161; A. Ste1lo, op. cit., p. 101.

19 La première édition: S. de Sacy, op. cit., p. 62-64; la nouvelle édition: E. Bas so, Il , bellum de Sorcati”..., p. 25-26; R. Grousset, op. cit., p. 514-523; B. Spuler, op. cit., p. 133-141; V. Ciocîltan, Mongoli..., p. 223-224; E. Basso, Il ,,bellum de Sorcati”..., p. 16-17, 17-18, 20.

20 ASG, MC, sala 34, 590/1225, fol. 6r (8.XI.1374). 
siège des autorités coloniales (in palacio comunis), le consul local accueille avec des confiseries, du vin et aliis similibus, forenses, indéfinis qui viennent ad vixitandum dominum consulem ${ }^{21}$. Étant donné que les documents de la Trésorerie ne notent pas d'autres aliments, l'on peut supposer que les confiseries et le vin avaient été les seuls aliments servis. Il y a lieu de souligner que les modalités d'accueil des hôtes avait été approuvées par le conseil local et l'office compétent dont le nom n'a pas été précisé (ut deliberatum fuit in consilio et per oficium) ${ }^{22}$. Une mention similaire est datée du mois de juin de la même année ${ }^{23}$.

Durant la période ultérieure, il y a d'autres informations similaires concernant les confiseries et le vin acquis par les autorités de Caffa pour accueillir des hôtes officiels. C'étaient aussi bien des députés du gouverneur de Solkhat que ses fonctionnaires venus prélever le droit de douane des colonies génoises ${ }^{24}$. La forme des inscriptions respectives concernant les circonstances dans lesquelles les confiseries étaient consommées permet de supposer que celles-ci n'étaient consommées avec du vin que lors des réceptions spéciales lors desquelles n'étaient servis que les aliments susmentionnés. Sûrement, ce n'étaient pas des festins de plusieurs heures - convivia, parce qu'il est clairement précisé dans les documents que les confiseries étaient acquises pro recipiendo ou pro tenendo coppam (de mense), ce qui est susceptible d'indiquer qu'il s'agissait d'amuse-bouches ${ }^{25}$. Ajoutons en marge qu'une situation analogue est observée à Pera (Galata) - quartier génois de Constantinople ${ }^{26}$.

En s'appuyant sur les arguments ci-dessus, l'on pourrait émettre - avec toute précaution - des hypothèses qui requièrent encore des vérifications. En premier lieu, les circonstances susmentionnées, dans lesquelles les confiseries étaient consommées peuvent être considérées comme celles qui, en Italie, étaient qualifiées de collatione dans la seconde moitié du XV $\mathrm{XV}^{\mathrm{e}} \mathrm{s}$. Dans les documents comparatifs, c'est-à-dire les documents de la Massaria de Caffa de la première moitié du XVe $\mathrm{S}$., ce terme apparaît à deux reprises (en $1423^{27}$ et en $1446^{28}$ ), toujours dans un contexte de relations diplomatiques. Ce fait permet d'admettre qu'une réception officielle avec du vin et de confiseries probablement faisait partie intégrante de la pratique diplomatique dans les relations entre les Génois et les représentants d'autres pays. En second lieu, l'on peut émettre l'hypothèse selon laquelle cette forme de réception était pratiquée dans le monde diplomatique génois sur la mer Noire depuis au moins 1375, mais d'autres termes étaient employés pour la désigner.

Outre les collationes, les confiseries étaient aussi probablement servies et mangées par les députés étrangers lors des festins organisés par les autorités de Caffa

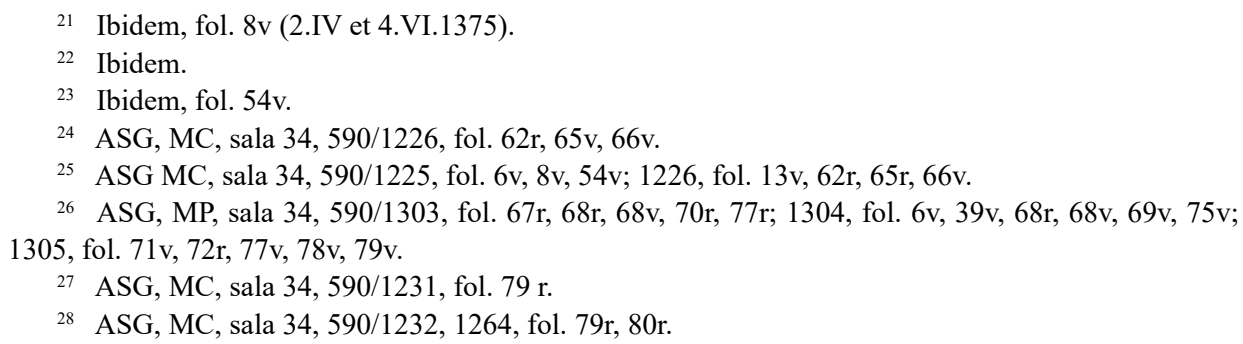


avec, en tête, le consul local. Les dimensions de ce phénomène sont très difficiles à étudier. Bien que le registre de la Massaria de Caffa du milieu des années $70 \mathrm{du}$ $\mathrm{XIV}^{\mathrm{e}} \mathrm{s}$. contienne des mentions sur les festins ou repas (convivium, pastum) organisés à l'occasion de la visite de Mamaï ou d'un de ses députés à Caffa (et fasse mention des montants dépensés à cette occasion), il ne précise jamais quels produits ont été achetés (y compris les confiseries qui nous intéressent). Précisons cependant qu'il découlerait des mentions qu'un festin (convivium) donné à l'occasion de la visite du gouverneur de Solkhat à Caffa était un événement habituel qui se déroulait au moins une fois par $a^{29}$.

Dans les années $80 \mathrm{du}$ XIV $\mathrm{e}$., la visite à Caffa de Ilias (Elias) Bey, gouverneur de la Crimée nommé par Tokhtamych, constitue le seul contexte qui permet d'associer les confiseries aux repas fastueux. Bien que la visite se déroule en août 1381, encore longtemps après la fin de celle-ci l'on trouve dans les documents de la Massaria des mentions sur les dépenses consenties pour différents produits de consommation (tels que: vin grec, volaille, différentes viandes sans plus de précisions, riz ou autres) et cadeaux (vêtements somptueux et tissus) ${ }^{30}$. En même temps, les autorités de Caffa ont acquis à l'un des speziarii locaux des confiseries, du safran, du sucre, de la cannelle, salsa (voir plus loin) et du sorbet consumptis in pasto facto dicto domino Elias-bey ut ordinatorum continetur ${ }^{31}$. (Peut-être que ce dernier terme se réfère aux visites annuelles des gouverneurs de Solkhat à Caffa et à l'accueil de ceux-ci avec un festin approprié).

Des confiseries sans plus de précisions ont aussi été servi en février 1382 lors d'un repas officiel organisé à l'occasion de la visite à Caffa des représentants du gouverneur de Solkhat qui prélevaient auprès des Génois un tribut mensuel (fixé par

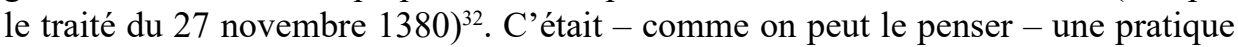
habituelle des Génois.

Les cadeaux diplomatiques constituent un autre domaine où apparaissent des mentions concernant les confiseries dans le contexte des relations diplomatiques de Caffa. Elles étaient offertes aux députés étrangers qui venaient à Caffa ou aux souverains tartares par l'intermédiaire des envoyés de Caffa. Comme il découle des études d'Annika Stello sur les objets offerts comme cadeaux diplomatiques de 1374 à 1446 par les autorités de Caffa, l'on peut dire que les confiseries constituaient l'un des types de cadeaux. Le plus souvent, les souverains tartares ou autres recevaient différents produits textiles: tissus ou produits finis. Au total, comme l'a calculé la chercheuse

${ }^{29}$ Quando venit pro faciendo convivium consuetum: ASG, MC, sala 34, 590/1225, fol. 6r; N. Iorga, op. cit., p. 32. Convivium sive pasto: ASG, MC, sala 34, 590/1225, fol. 6; N. Iorga, op. cit., p. 32. Pastum filie domini de Surchati: ASG, MC, sala 34, 590/1225, fol. 56r; N. Iorga, op. cit., p. 34. En outre: ASG, MC, sala 34, 590/1225, fol. 55r; N. Iorga, op. cit., p. 34. Les frais pour le séjour a Caffa des ambassadeurs tartres et répresentant du prince de Teodoros-Mangup: ASG, MC, sala 34, 590/1227, fol. 27; N. Iorga, op. cit., p. 43; ASG, MC, sala 34, 590/1229, fol. 53, 76v; N. Iorga, op. cit., 49 (7.IX.1422); En outre ont été mentionnés les le dépenses in convivio facto Solimano Sarraceno misse parte dominorum Surchatensium ut moris est: ASG, MC, sala 34, 590/1230, fol. 32v; N. Iorga, op. cit., p. 49.

30 Pour le prix de 1134 aspres: ASG, MC, sala 34, 590/1226, fol. 294, N. Iorga, op. cit., p. 42.

31 ASG, MC, sala 34, 590/1226, fol. 294; N. Iorga, op. cit., p. 42.

32 ASG, MC, sala 34, 590/1226, fol. 294v; N. Iorga, op. cit., p. 43. 
allemande, durant la période considérée les dons de ce type ont été mentionnés 576 fois. Pendant cette période on mentionne les confiseries comme dons seulement à 18 reprises et, au XIV $\mathrm{e}$ s., uniquement 4 fois $^{33}$.

Après avoir étudié les circonstances à l'occasion desquelles les confiseries étaient consommées, essayons de répondre à la question de savoir quelles confiseries étaient servies lors des réceptions diplomatiques à Caffa en Crimée ou offertes aux souverains étrangers ou à leurs envoyés?

Dans la majorité des cas les sources génoises utilisent les termes généralux de confecta vel confectes. Et ici se pose une autre question: qu'est-ce qui se cache sous ces termes?

La littérature italienne concernant cette problématique (C. Benporat ${ }^{34}, \mathrm{E}$. Carnevale Schianca ${ }^{35}$ ) met en exergue la bipolarité de la signification de ce terme. Au sens étroit, ces termes s'appliquaient à tout produit confectionné, composé d'une garniture enrobée de sucre. Ainsi, la garniture pouvait être constituée de grains séparés (par ex.: de coriandre ou d'anis) et d'épices entiers ou en morceaux (par ex. cannelle, cardamome, fleur de muscade (macis), gingembre, noix de muscade etc.), ainsi que d'amandes et de différentes variétés de noix. Au sens large, ces termes définissaient des produits confits (canditi) et des gelées (gelatine) ${ }^{36}$.

Dans le contexte qui nous intéresse, il est difficile de dire de manière univoque à quel domaine de signification se réfère le terme confectes. Peut-être s'agit-il d'un terme qui englobe toutes les confiseries indépendamment de leur consistance. (Al'appui de cette thèse l'on peut soulever que, dans les sources étudiées, il n'y a pas de terme electuaria s'appliquant aux confitures ou aux fruits confits).

Le sorbet figure parmi d'autres sucreries ou plutôt plats de dessert, mentionnés dans les documents de la Massaria de Caffa. De même que selbeto, il apparaît dans le contexte des achats effectués à l'occasion de la visite à Caffa du gouverneur de Solkhat, Elias bey, en août 1381. Cerbeto pro nutrimento a été servi aux députés ottomans qui sont venus à Caffa en novembre $1410^{37}$.

Dans le contexte des confiseries achetées chez l'épicier local est aussi employé un autre terme-salsa. Peut-être sous ce terme se cache-t-il salsa confetta, dont la recette est donnée dans le manuscrit génois Medicinalia quam plurima du XV $\mathrm{X}$. Conformément aux indications, il s'agit d'une mixture faite d'amandes râpées (4 livres), raisins râpés (2 livres) avec différentes épices (éventuellement avec du sel), y compris avec du safran et d'une grande quantité de miel (10 livres) $)^{38}$. On ne peut pas exclure non plus qu'il s'agisse d'un mélange d'épices ordinaires; évidemment, il n'est pas aisé de trancher la question de manière univoque.

${ }_{33}$ A. Stello, op. cit., tab. 2, p. 150.

34 C. Benporat, Note per la storia dei confetti, „Appunti di gastronomia” 2005, 47, p. 71-72.

35 E. Carnevale Schianca, La cucina medievale. Lessico, storia, preparazioni, Firenze 2011, p. 178; G. Rebora, La cucina medievale italiana tra Oriente ed Occidente [dans:] Miscellanea storica ligure, Genova 1990, p. 1542-1543.

36 E. Carnevale Schianca, op. cit., p. 178-179.

37 N. Iorga, op. cit., p. 47, note 3 (18.IX.1410).

38 E. Carnevale Schianca, op. cit., p. 579, 582. 
Il est sûr que la liste des types énumérés ne constitue pas une liste exhaustive des confiseries utilisées dans la diplomatie génoise en Crimée à la fin du XIV ${ }^{e} s$. De plus, comme je l'ai déjà signalé, parfois l'identification ne se fonde que sur des suppositions; l'éclaircissement de cette problématique requiert encore beaucoup d'études.

En résumant les considérations, on peut formuler les conclusions présentées dans les points qui suivent.

En premier lieu, dans les colonies génoises dont l'existence était fondée sur les vastes relations commerciales, le sucre et ses différentes formes transformées, c'està-dire les confiseries, étaient l'une des marchandises disponibles. Bien que, pendant des siècles, les confiseries passaient pour des produits médicinaux, à une époque difficile à déterminer, elles sont parvenues sur les tables comme produits pharmaceutiques consommés pour le plaisir. Par ce fait extraordinaire, depuis le dernier quart du XIV s. les confiseries sont présentes dans la diplomatie génoise sur la Mer Noire; elles ont été le plus fréquemment consommées comme amuse-gueules qui-comme en peut s'en douter - accompagnaient les négociations diplomatiques, mais étaient plus rarement servies lors des festins fastueux.

En second lieu, les confiseries étaient aussi offertes comme dons. Au XIV ${ }^{e} \mathrm{~s}$. c'était une pratique relativement rare dans la diplomatie génoise. Elle pouvait dépendre des goûts des destinataires et, peut-être, de la mode ou de la pratique locale. Les souverains tartares de la Crimée et leurs représentants avaient une prédilection pour les objets matériels durables. Durant la première moitié du $\mathrm{XV}^{\mathrm{e}}$ s., le rôle des confiseries comme dons marque une tendance à la hausse. Peut-être était-ce dû au fait que, tout doucement, les destinataires tartares de ces dons se familiarisaient avec des formes de cadeau plus raffinées.

En troisième lieu, dans la perspective présentée ci-dessus, on peut dire que les confiseries ne jouaient pas un rôle essentiel dans la diplomatie mais, dans l'entourage complexe du monde de la diplomatie du bas Moyen-Âge, elles constituaient l'un de ses éléments. Tout simplement, elles constituaient son complément sucré.

\section{BIBLIOGRAPHIE}

\section{Sources manuscrites}

Archivio di Stato di Genova, Banco di San Giorgio, Massaria Caffae, sala 34, 590/1225, 1226, 1226-1, 1227-1235, 1264.

Archivio di Stato di Genova, Banco di San Giorgio, Massaria Peire, sala 34, 590/1303-1305.

\section{Sources imprimées}

Balducci Pegolotti F., La pratica della mercatura, ed. A. Evans, Cambridge, Mass. 1936. 
Desimoni C., Trattato dei Genovesi col chan dei Tartari nel 1380-1381, scritto in lingua volgare, „Archivio Storico Italiano” 20, 1887, p. 161-165.

Iorga N., Notes et extraits pour servir à l'histoire des croisades au XV siècle, „Revue de l'Orient latin" 1896, vol. IV, p. 25-118.

Sacy S. de, Pièces diplomatiques tirées des Archives de la Républiques du Gênes [dans:] Notices et extraits des manuscrits de la Bibliothèque du Roi, vol. XI, Paris 1827, p. 1-91.

\section{Publications}

Ashtor E., Il volume del commercio levantino di Genova nel secondo Trecento [dans:] Civico Istituto Colombiano, Studi e Testi, p. 2, Serie storica a cura di G. Pistarino, Saggi e documenti, vol. I, Genova 1978, p. 391-432.

Balard M., Gênes et la mer Noire (XIII $-X V^{e}$ siècle), „Revue Historique” 270, 1983, p. 31 40.

Balard M., Les Génois et les régions bulgares au XIV siècle, „Byzantino-bulgarica” 1981, 7, p. 87-97.

Balard M., Precursori di Cristoforo Colombo: i Genovesi in Estremo Oriente nel XIV secolo [dans:] Atti del Convegno Internazionale di Studi Colombiani (Genova 13-14 ottobre 1973), Genova 1974, p. 149-161.

Balard M., La Romanie génoise (XII - début du XV siècle), vol. I-II, Roma-Genova 1978.

Basso E., „De rebus castri Illicis et alia”. Genova, la Moldavia e la Valacchia fra cooperazione e contrasto nel secondo Quattrocento [dans:] Italia e Romania. Due popoli e due storie a confronto (secc. XIV-XVIII), a cura di S. Graciotti, Firenze 1997, p. 83-98.

Basso E., Il „, bellum de Sorcati” ed i trattati del 1380-87 tra Genova e l'Orda d'Oro, „Studi Genuensi" 1990, n. s., 8, p. 11-24.

Bautier R.H., Les relations économiques des Occidentaux avec les pays d'Orient au Moyen Age. Points de vue et documents [dans:] Sociétés et compagnies de commerce en Orient et dans l'Océan Indien, Actes du 8 Colloque international d'Histoire maritime, Beyrouth 1966, Paris 1970, p. 263-310.

Benporat C., Note per la storia dei confetti, „Appunti di gastronomia” 2005, 47, p. 71-89.

Carnevale Schianca E., La cucina medievale. Lessico, storia, preparazioni, Firenze 2011.

Ciocîltan V., Mongoli şi Marea Neagră în secolele XIII-XIV. Contribuţia Cinghizhanizilor la transformarea bazinului pontic în placă turnantă a comerţului euro-asiatic, Bucureşti 1998.

Ciocîltan V., Reichspolitik und Handel: Die tatarisch-genuesischen Verträge von 1380 1387, „Il Mar Nero. Annuario di archeologia e storia” 1994, 1, p. 261-278.

Ciocîltan V., Restauraţia Hoardei de Aur şi tratatele tătaro-genovese din anii 1380-1387, „Revistă Istorică” 1990, 1, n. 6, p. 571-595.

Grekow B., Jakubowski A., Złota Orda i jej upadek, Warszawa 1953.

Grousset R., L'Empire des steppes, Paris 1939.

Heyd W., L'histoire du commerce du Levant au Moyen Age, vol. I-II, Paris 1936.

Hryszko R., Z Genui nad Morze Czarne. Z kart genueńskiej obecności gospodarczej na pótnocno-zachodnich wybrzeżach Morza Czarnego u schyłku średniowiecza, Kraków 2004.

Jehel G., Les Génois én Mediterranée occidentale (fin XI'-début XIV siècle), Paris 1993.

Karpov S.P., Ital'janskie morskie respubliki i Juznoe Pricernomor'e v XIII-XV vv.: problemy torgovli, Moskva 1990. 
Kedar B.Z., Mercanti in crisi: Venezia e Genova, Roma 1981.

Khvalkov E., The Colonies of Genoa in the Black Sea Region: Evolution and Transformation, New York-London 2017.

Lopez R.S., In quibuscumque mondi partibus [dans:] Miscellanea di storia italiana e mediterranea per Nino Lamboglia, Genova 1978, p. 345-354.

Lopez R.S., L'extrême frontière du commerce medieval, „Le Moyen Âge” vol. jubilaire: 1963, p. 479-490.

Lopez R.S., Storia delle colonie genovesi nel Mediterraneo, Genova 1996.

Małowist M., Kaffa - kolonia genueńska na Krymie i problem wschodni w latach 14531475, Warszawa 1947.

Mavrina O.S., Nekotoryye aspekty genuezsko-tatarskikh otnosheniya v XIV veke, „Skhodoznavstvo" 2005, 29-30, p. 89-99.

Nasov A.N., Mongoly i Rus, Moskva-Leningrad 1940.

Papacostea Ş., „, Quod non iretur ad Tanam”. Un aspect fondamental de la politique génoise dans la mer Noire au XIV siècle, „Revue des Études Sud-Est Européenes” 1979, 17, n. 2, p. 201-217.

Pilipchuk Ya.V., Voyny v zolotoordynskom Krymu: realii i vymysel (40-ye gg. XIV v-10-ye gg. $X V$ v.), „Parabellum novum” 2017, nr 7(40), p. 55-69.

Pioro I.S., Krymskaja Gotija: Očerki etničeskoj istorii naselenija Kryma v pozdnerimskij period i rannee srednevekovie, Kiev 1990.

Pistarino G., I Gin dell'Oltremare [dans:] Civico Istituto Colombiano, Studi e Testi, Serie storica a cura di G. Pistarino, Genova 1988.

Ponomarev A.L., Putevoditel' po rukopisi massarii Kaffy 1374 g. Liber massariae Caffae tempore regiminis egregii viri domini Iuliani de Castro consulis Caffae MCCCLXXIV nunc indicatus et a pluribus mendis purgatus [dans:] Prichernomorie $v$ srednie veka, vypusk 6, Moskva 2005, p. 41-138.

Ponomarev A.L., «Solkhatskaya voyna» i «imperator» Bek Bulat [dans:] Materialy vtoroi Mezhdunarodnoy nauchnoy konferentsii «Politicheskaya i sotsial'no-ekonomicheskaya istoriya Zolotoy Ordy», posvyashchennoy pamiati M.A. Usmanova, Kazan', 29-30 marta 2011 g., Kazan' 2011, p. 18-21.

Quirini-Popławska D., Włoski handel czarnomorskimi niewolnikami w późnym średniowieczu, Kraków 2002.

Rebora G., La cucina medievale italiana tra Oriente ed Occidente [dans:] Miscellanea storica ligure, Genova 1990, p. 1530-1560.

Spuler B., Die Goldene Horde. Die Mongolien in Russland (1223-1502), Wiesbaden 1965.

Stello A., Grenzerfahrung. Interaktion und Kooperation im spätmittelalterlichen Schwarzmeerraum, Karlsruhe 2012, http://ubt.opus.hbz-nrw.de/volltexte/2012/753 [accès: 8.09.2017].

Vasiliev A.A., The Goths in the Crimea, Cambridge 1936. 